\title{
Unique method for human villous trophoblasts isolation from placental tissue explants
}

\author{
Ashley Serjilus ${ }^{1}$ and Donald J Alcendor ${ }^{2 *}$ \\ ${ }^{1}$ Department of Obstetrics and Gynecology, School of Medicine, Meharry Medical College, USA \\ ${ }^{2}$ Center for AIDS Health Disparities Research, Department of Microbiology, Immunology, and Physiology, Meharry Medical College, School of Medicine, Nash- \\ ville, TN, USA
}

\begin{abstract}
Isolation of cytotrophoblasts from primary placental tissue may be costly and time consuming with variable results. In this paper, we provide a simple, affordable, and efficient method that may performed using common laboratory supplies to achieve consistent in vitro isolation of cytotrophoblasts from villous tissue. Trophoblast populations are identified based on morphology and phenotyping, which employs the timely extraction of villous nodes from the placenta prior to cultivation and isolation of nodal outgrowth by visual guidance for selective capture of cytotrophoblast populations and subculture. This method allows for the isolation of cytotrophoblasts free of contamination with other placental cell types. Isolated cells stain positive for the specific cytotrophoblast biomarker cytokeratin 7 and Human Chorionic Gonadotropin (HCG). Subcultured cells grow to confluency to establish monolayers that may be passaged in culture and later used to develop primary syncytiotrophoblasts over time. These primary cytotrophoblast populations may be employed using in in vitro placenta-on-a chip models to better understand placental cell biology and function, as well as physiological responses after exposure to toxicants, and infectious agents. This technique may be modified for selective isolation of specific cell types within different tissues from multiple organ systems.
\end{abstract}

\section{Introduction}

Conventional cell culture techniques that use different types of placenta-derived cells to understand placenta biology have been useful but lack organ-specific ultrastructure and physiological function of the placenta. Modeling of the human placenta to better understand placenta biology in human development has culminated into the establishment of placenta-on-a-chip models based on the compartmentalization of primary human placenta cells in vitro that can be monitored in real time [1-4]. These microdevices are created by using microfluidics and microfabrication technologies and microsystems consist of two polydimethylsiloxane (PDMS) microfluidic channels separated by a thin extracellular matrix (ECM) membrane [2]. Essential cellular components of these model placenta microsystems are placental trophoblasts.

The widespread use of commercially available trophoblast cell lines, such as BeWo, JEG-3, or JAR, that are derived from human choriocarcinoma, have been useful. However, these transformed cell lines, some of which have been in culture for decades, do not mimic primary trophoblasts in vivo [5-8]. The task of having to isolate primary cytotrophoblasts from placental tissue may be laborintensive and require expensive reagents with variable results. Routine villous trophoblasts isolation based on trypsin digestion of placental villi, followed by additional purification steps, may be costly and labor-intensive [9]. Purification of villous trophoblasts using Percoll gradients was shown to yield a purity of about $80 \%$ by Kilman et al. [9-11]. Magnetic beads also have been used to further purify villous trophoblasts from trypsin- treated placental explants [12,13]. Other groups have demonstrated the separation of cytotrophoblasts from mononucleated syncytial fragments [14-16]. However, these procedures still required second-line separation technologies to achieve reasonable purity from other contaminating placental cell types. In this paper, we describe the procedure use to isolate purified villous cytotrophoblasts from term placental explants. This procedure involves the extraction of villous nodes from human placental tissue, continuous cultivation in trophoblasts media, and visual guidance for selective capture by clonal selection of cytotrophoblast populations, subculture, and validation of cytotrophoblast populations for identity and purity [17].

\section{Materials and methods}

\section{Placenta collection}

Placentas were obtained from elective non-laboring caesarean sections after uncomplicated full-term pregnancies at Vanderbilt University Medical Center. These studies were approved by the Vanderbilt University Institutional Review Board.

\section{Trophoblasts isolation}

All procedures involved in the collection of placentas and placental explants and their transport were performed under sterile conditions. The time from delivery to placental node isolation did not exceed 1 hour. For cytotrophoblasts isolation, placental nodes or cotyledons were washed three times with phosphate buffered saline (PBS) pH7.4

${ }^{*}$ Correspondence to: Donald J Alcendor, Associate Professor of Microbiology and Immunology, Center for AIDS Health Disparities Research, Meharry Medical College, 1005 Dr. D.B. Todd Jr. Blvd., Hubbard Hospital, 5th Floor, Rm. 5025, Nashville, TN 37208, USA, E-mail: dalcendor@mmc.edu

Key words: placenta, trophoblasts, cytotrophoblasts, explants, isolation, cultivation

Received: October 19, 2020; Accepted: November 04, 2020; Published: November 10, 2020 
and excised with sterile surgical scissors, and the decidual layer was removed to expose villous tissue. Placental nodes/cotyledons were minced with a number 21 scalpel, and individual 5-mm tissue explants were spatially added to $100 \times 20-\mathrm{mm}$ dishes and cultured in $10 \mathrm{mls}$ of trophoblast medium per plate (10\% fetal bovine serum, $1 \%$ penicillin/ streptomycin; ScienCell, Carlsbad, CA) [17]. Trophoblast medium was supplemented with $25 \mathrm{ug} / \mathrm{ml}$ of Fungizone (Gibco, Life Technologies, Grand Island, NY). Trophoblast explant outgrowth was examined daily by microscopy. Trophoblast colonies are identified using an inverted microscope. Distinct colonies were identified by morphology and encircled using a felt tip marker prior to extraction. Colony extraction was accomplished using the barrel of a plastic, sterile 1-ml pipette tip after the tip was removed with the scalpel. Media was removed from the dish, the open barrel of the pipette tip is dipped in sterile Vaseline, and the end of the pipette barrel containing Vaseline was placed directly over the trophoblast colony on the plate as the Vaseline created a seal around the cell colony. Trypsin is added (0.05\% trypsin/EDTA (Gibco, Life Technologies, Grand Island, NY) to the open end of the pipette barrel for $10 \mathrm{~min}$ at $37^{\circ} \mathrm{C}$. Trypsinized cytotrophoblasts were added to 4 -well chamber slides containing trophoblast medium. Confluent cells were confirmed as trophoblasts by staining with the epithelial intermediate filament antigenic biomarker cytokeratin 7 (Millipore, Bedford, MA).

\section{Cells and viruses}

The HCMV-GFP recombinant virus expressing green fluorescent protein was obtained from Dr. Gary Hayward, Johns Hopkins University. HCMV-GFP was cultivated in human foreskin fibroblasts at a multiplicity of infection (moi) of 01 . Viral titers were determined by limiting dilution using a fluorescent focus assay [18]. All infections with the SBCMV clinical strain were performed at passage level 3 [1819]. We are aware that high-level passage in vitro may result in the acquisition of mutations that may impact viral tropism and replication.

\section{HCMV-GFP infection of Placental histocultures}

Approximately 20 grams of minced villous tissue were added to $30 \mathrm{ml}$ of trophophoblast media in a $50-\mathrm{ml}$ conical tube prewarmed to $37^{\circ} \mathrm{C}$. The HCMV-GFP recombinant virus at a moi 0.1 was used to infect trophoblasts histocultures and was incubated for 1 hour at $37^{\circ} \mathrm{C}$ [17]. After 1- hour post-infection the media was removed, and fresh media was added. Next, the histocultures were incubated at $37^{\circ} \mathrm{C}$ for 96 hours. Infected villous tissue was placed in chamber slides and examine by fluorescent microscopy for GFP expression.

\section{Immunofluorescence}

Immunohistochemistry was performed as previously published (Chamber slide cultures containing subcultured trophoblasts were washed twice with PBS ( $\mathrm{pH} 7.4$ ), air-dried, and fixed at $-20^{\circ} \mathrm{C}$ in absolute methanol for 10 minutes. Cells were air-dried for 15 minutes, hydrated in Tris-buffered saline ( $\mathrm{pH}$ 7.4) for $5 \mathrm{~min}$, and incubated separately for 1 hour with mouse monoclonal antibodies against cytokeratin 7, (Santa Cruz Biotechnology, Dallas TX), diluted 1:50 in PBS (pH 7.4). Cells were washed three times with Tris saline and then incubated at $37^{\circ} \mathrm{C}$ for 30 minutes with a combination of secondary donkey anti-mouse Immunoglobulin $\mathrm{G}$ (IgG) antibodies, conjugated with fluroescein isothiocyanate (FITC), (Jackson ImmunoResearch, West Grove, PA) at a 1:100 dilution in PBS $[18,19]$. Cells were washed another three times in Tris saline and mounted with Vectashield mounting media (Vector Laboratories, Burlingame, CA) containing $1.5 \mu \mathrm{g} / \mathrm{ml}$ of 4',6-diamidino2-phenylindole (DAPI). Fluorescence was photographed with a Nikon
TE $2000 \mathrm{~S}$ fluorescent microscope mounted with a charge-coupled device (CCD) camera (Nikon, Tokyo, Japan).

\section{Immunohistochemistry}

Primary trophoblasts were cultivated in chamber slides at a density of $1 \times 10^{4}$ cells/well in trophoblasts media with and without forskolin for 72 hours. Cells were wash $3 \mathrm{X}$ with $\mathrm{PBS} \mathrm{pH} 7.4$, air dried at room temperature, and fixed in $100 \%$ methanol at $-20 \mathrm{C}$ for 30 minutes. Cells were then air dried, hydrated in PBS, and immunohistochemistry (IHC) was performed as previously described [19] using a mouse monoclonal antibody to Human chorionic gonadotropin (HCG). DAB was used as a peroxidase substrate for color development. Positive trophoblasts appear brown in color.

\section{Forskolin treatment}

Primary trophoblasts were cultivated in chamber slides at a density of $1 \times 104$ cells/well in trophoblasts media and treated with $25 \mu \mathrm{M}$ of forskolin (Sigma-Aldrich Inc.) for 72 hours. Mock treated cells were given media only.

\section{Results}

\section{Explant cultivation}

Nodes are collected from intact placentas under sterile conditions. Villous nodes were first submerged in complete trophoblast media and transported on ice. Before dissection, nodes were washed in an excess of PBS to reduce erythrocyte contamination and hydrate the tissue (Figure 1). Villous nodes are sectioned with a scalpel and surgical scissors and placed in dishes. Fresh, prewarmed trophoblast media was added, and explant cultures were incubated at $37 \mathrm{C}$ for $1-16$ days, with fresh media changes every 2 days.

\section{Placental histocultures support HCMV-GFP replication}

In a previous publication, we determined that villous trophoblasts are highly permissive for HCMV lytic replication. To determine if there were viable cells that could support replication of HCMV, we infected histocultures of villous nodes with a recombinant HCMV-GFP. Villous nodes are known to be enriched with villous cytotrophoblasts, and knowing their permissiveness for HCMV, we would expect GFP expression only in cells that could support HCMV replication (Figure 2). At 96 hours post-infection, we observed GFP-positive cells by fluorescent microscopy (Figure 2) in peripheral tissue, isolated cells (Figure 2A, 2B, and 2C), and internal villous tree structures (Figure 2D). We also observed a significant amount of erythrocyte contamination in all specimens examined (Figure 2).

\section{Nodal outgrowth of cytotrophoblasts}

An illustration of the cultivation process for isolation of trophoblasts is shown in Figure 3. We cultured villous explants in $100 \times 20 \mathrm{~mm}$ dishes in $10 \mathrm{ml}$ of trophoblast medium after trypsinization for isolation of villous trophoblasts over the course 16 days (Figure 3). Fresh media was added every 2 days during the cultivation period. On day 1 , explants appeared aggregated, and some were adherent (Figure 4A). At 8 days post-cultivation I observed small colonies of cells with characteristic trophoblast morphology (Figure 4B). On day 11, we observed a significant expansion of these colonies that remained subconfluent (Figure 4C). After 14 days, we observed patches of confluent growth with trophoblasts morphology (Figure 4D). These cells were round or oval, or was multipolar, elongated cell monolayer previously described as "crazy pavement" [20-23]. In addition, we observed what appeared as 

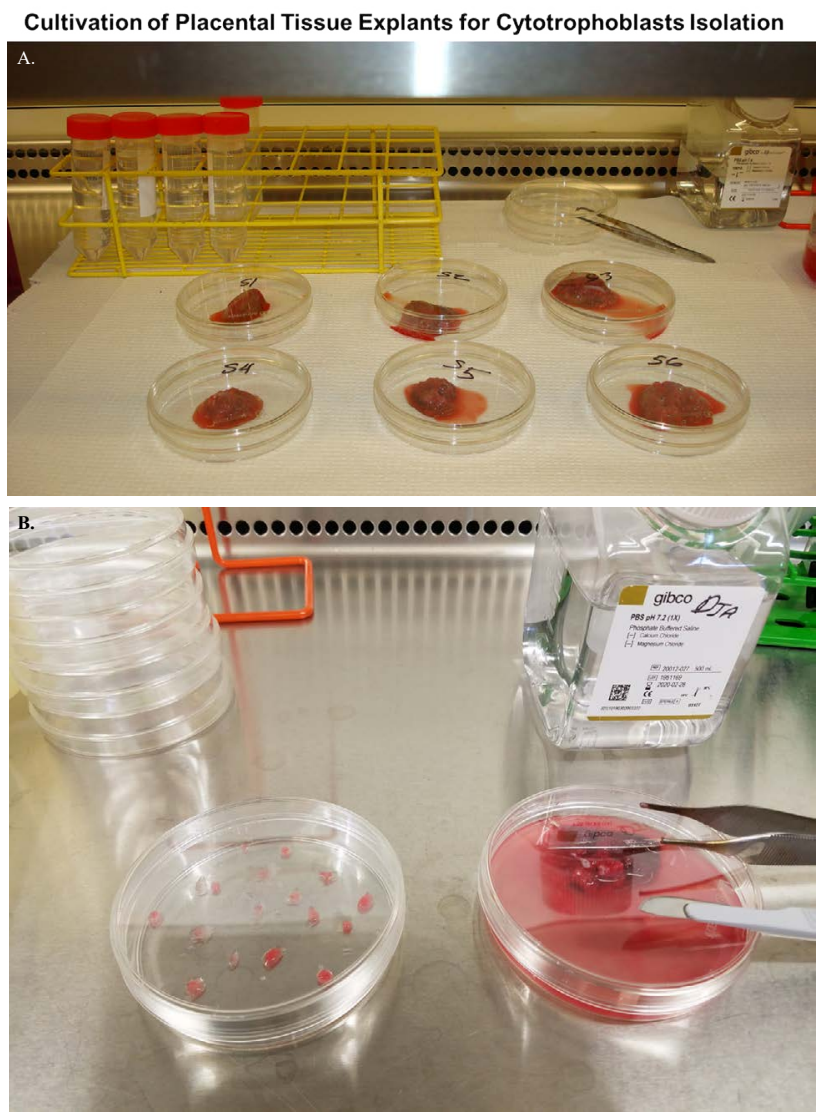

Figure 1. Cultivation of placenta explants for cytotrophoblast isolation. Villous nodes/cotyledons are excised from placentas with a scalpel and surgical scissors in a laminar flow hood and transported on ice in $50 \mathrm{ml}$ conical tubes containing $30 \mathrm{ml}$ trophoblasts media. Nodes are soaked in access PBS pH7.4 prior to dissection and cultivation

Placental Explant Histocultures Infected with HCMV-GFP 96 hours Post Infection
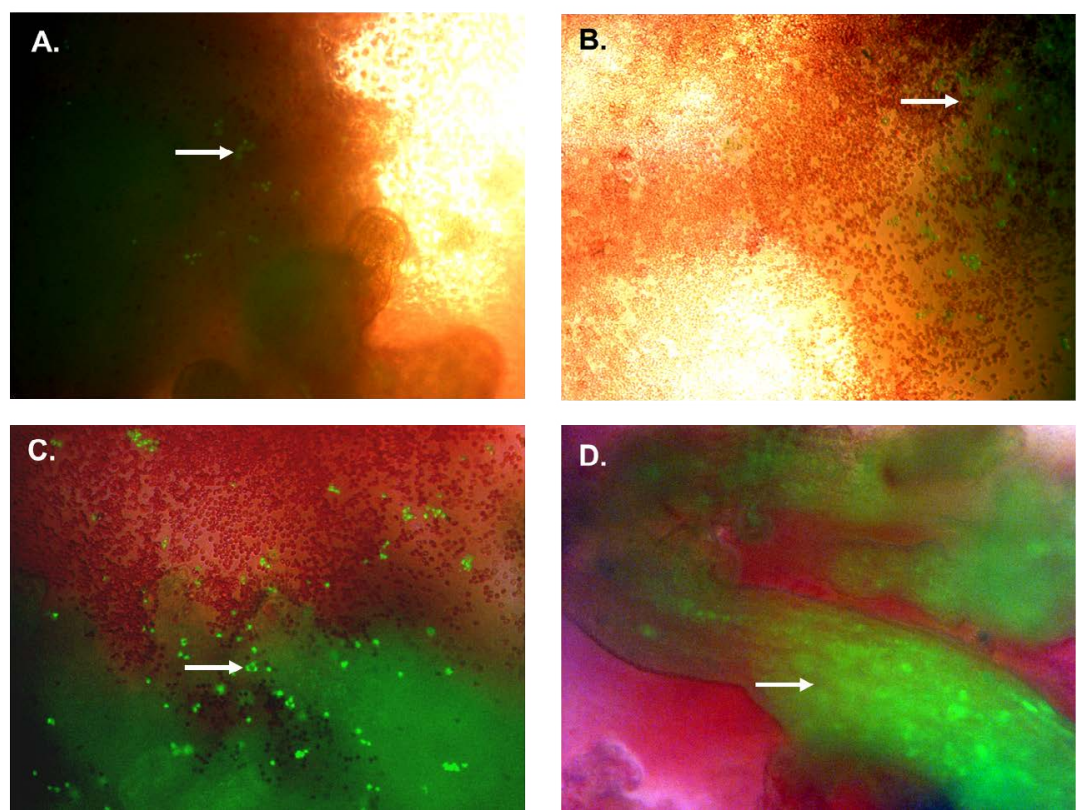

Figure 2. Placental explant histocultures infected with HCMV-GFP. Placental explant histocultures were infected with HCMV-GFP and examined for GFP fluorescence 96 hours postinfection. (A). Fluorescent microscopy of viable cells infected with HCMV-GFP expressing green fluorescent protein in peripheral placental tissue as shown by the white arrow. (B). Fluorescent microscopy of viable cells infected with HCMV-GFP expressing green fluorescent protein in peripheral placental tissue and contamination with erythrocytes as shown by the white arrow. (C). Fluorescent microscopy of viable cells infected with HCMV-GFP expressing green fluorescent protein in aggregate issue and contamination with erythrocytes as shown by the white arrow. (D). Fluorescent microscopy of viable cells in villous tree structures infected HCMV-GFP expressing green fluorescent protein as shown by the white arrow. All images were obtained using a Nikon TE2000S microscope mounted with a charge-coupled device (CCD) camera at $\times 200$ magnification 
A.

Trophoblasts colonies after 16 days cultivation of placenta explants in trophoblasts media

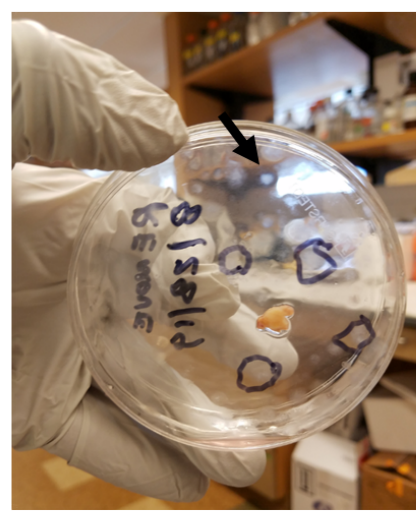

B. Isolation of Cytotrophoblasts from human placenta villous explants

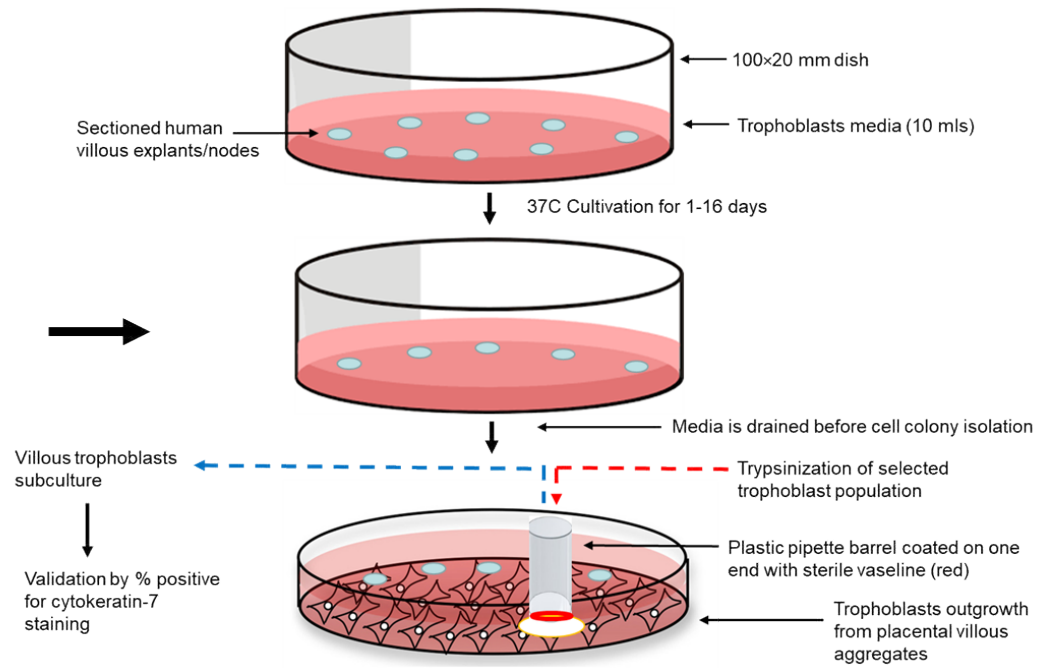

Figure 3. Scheme for isolation of cytotrophoblasts from placental explants. The isolation of trophoblasts involves the sectioning and placement of villous explants on a petri dish and cultivation and isolation of selected cell colonies from outgrowth from villous aggregates using a simple pipette barrel coated with Vaseline, followed by trypsinization and subculture to access purity by cytokeratin 7 staining

Trophoblasts Isolation from at Term Placenta Villous Explants after Trypsinization
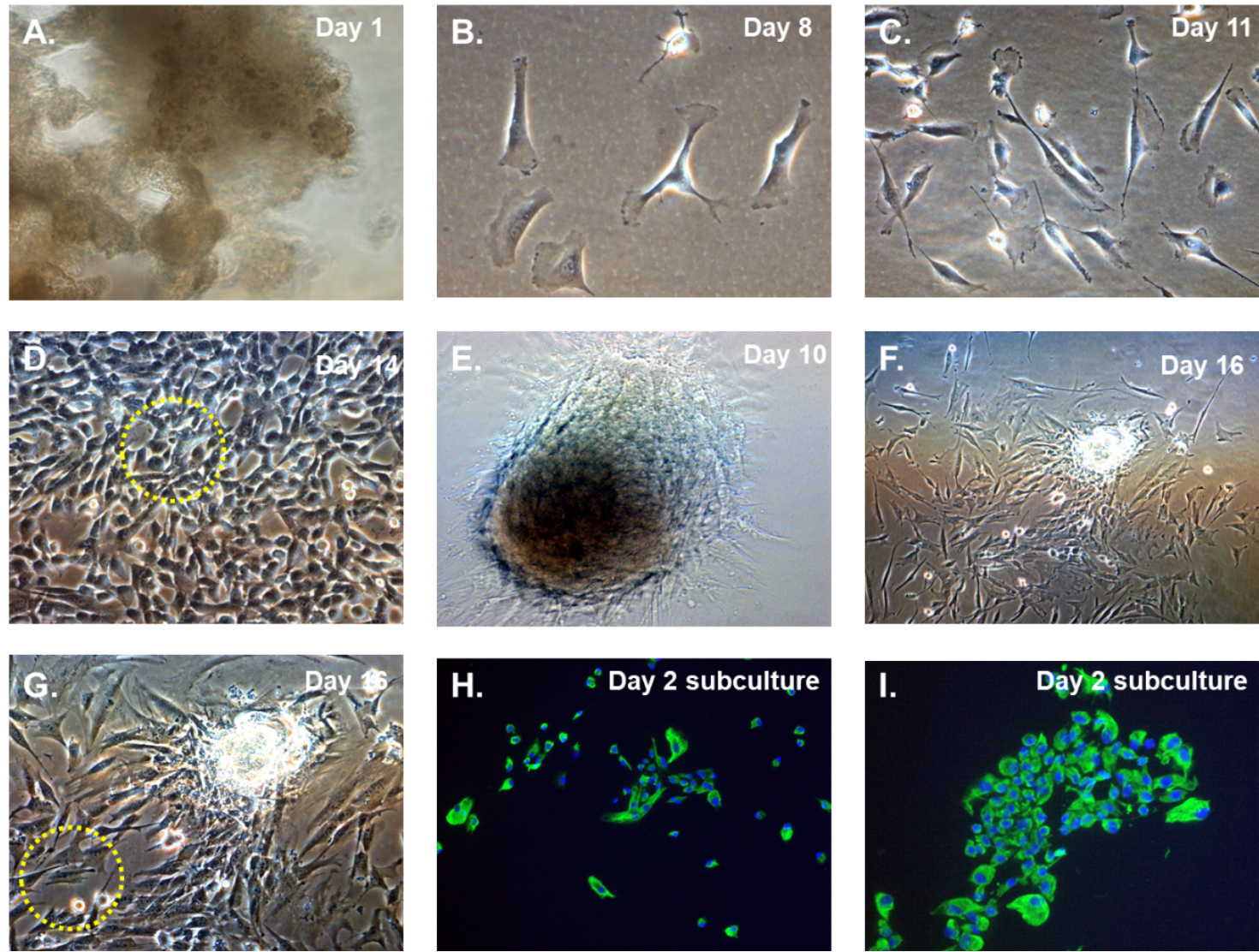

Figure 4. Trophoblasts isolation from at-term placenta villous explants after trypsinization. Time course isolation of trophoblasts from at-term placenta villous explants after trypsinization (A) placental nodes/cotyledons 24 hours after excision and dissection cultivated in trophoblasts media; (B) Villous cell outgrowth and early colony formation 8 days post-cultivation; (C) Villous cell outgrowth and subconfluent colony formation 11 days post-cultivation; (D) Villous cell outgrowth and confluent focal monolayers 14 days post-cultivation with yellow-dotted outlined areas designated for clonal selection and expansion; (E) Villous tissue aggregates in culture at 10 post-cultivation (F) Villous tissue aggregates and associated villous cell outgrowth 16 days post-cultivation (G) Higher magnification of villous tissue aggregates and associated villous cell outgrowth 16 days post-cultivation with yellow dotted outlined areas designated for clonal selection and expansion; (H) Subculture and cultivation of clonally selected cells that stain positive for cytokeratin 7 by immunofluorescent staining after 24 hours; (I) Subculture and cultivation of clonally selected cells that stain positive for cytokeratin 7 by immunofluorescent staining after 48 hours. For immunostained images, nuclei were stained blue with 4',6-diamidino-2-phenylindole (DAPI). All images were obtained using a Nikon TE2000S microscope mounted with a charge-coupled device (CCD) camera at $\times 200$ magnification 
trophoblasts aggregates 10 days post-cultivation (Figure 4E). At day 16, we observed significant cell outgrowth from these villous trophoblasts aggregates (Figure 4F). After examination, we observed that these cells had features consistent with villous trophoblasts.

\section{Selective capture and subculture of purified cytotrophoblast populations}

Nodal outgrowth of satellite cells that developed into confluent mini-colonies, which became subconfluent over time (Figure 4D), and cell outgrowth obtained from nodal aggregates (Figures $4 \mathrm{~F}$ and $4 \mathrm{G}$ ) were preferred for selective capture and subculture. These colonies appeared more homogenous and had morphological characteristics and growth rates consistent with trophoblasts monolayers. Several colonies were selected using the trypsin-pipette-Vaseline method of isolation, as described in the Materials and Methods section (Figure 3). Trypsinized cells from colonies were subcultured in duplicate chamber slides for both cell expansion and validation by immunofluorescent staining (Figure 3).

\section{Validation of trophoblast isolation}

After 48 hours, we observed that subcultured, subconfluent cell populations were viable, adherent, and stained positive for the trophoblast antigenic biomarker cytokertin 7 (Figure $4 \mathrm{H}$ and 4I). After cells became confluent, I observed universal cytokeratin 7 staining of purified cytotrophoblasts by fluorescent microscopy (Figures 5A-5D). Primary trophoblasts were cultivated in chamber slides at a density of $1 \times 104$ cells/well in trophoblasts media with and without forskolin for 72 hours. Cells treated forskolin had higher levels of HCG as determined by IHC staining (Figures 6A-6D).

\section{Discussions}

Several methods exist for isolating trophoblasts from placental tissue. In this study, we explore the cultivation of villous trophoblasts from placental nodes/cotyledons. The trypsin-pipette-Vaseline method includes Vaseline to seal the edges of the pipette barrel for focal trypinization. Creating the seal using Vaseline prevent crosscontamination by floating cells or cells from distal colonies that can occur when using cloning cylinders (Figure 2) [24]. We are aware of the similarities that exist among the different methods for isolation of trophoblast populations involving standard trypsinization; however, we employed a simple, affordable, and efficient for method that may be performed with common laboratory supplies to achieve consistent in vitro isolation of purified trophoblasts from placental villous tissue. Selected colonies are first identified during early stages of colony formation as shown by figure 4B. The colonies when identified are followed in development by microscopy until it reaches visual size for selection. The selection process only continues if the subcultured cells stain positive for the universal trophoblasts marker cytokeratin-7

\section{IFA Staining of Human Villous Trophoblasts Isolated from Placental Tissue}
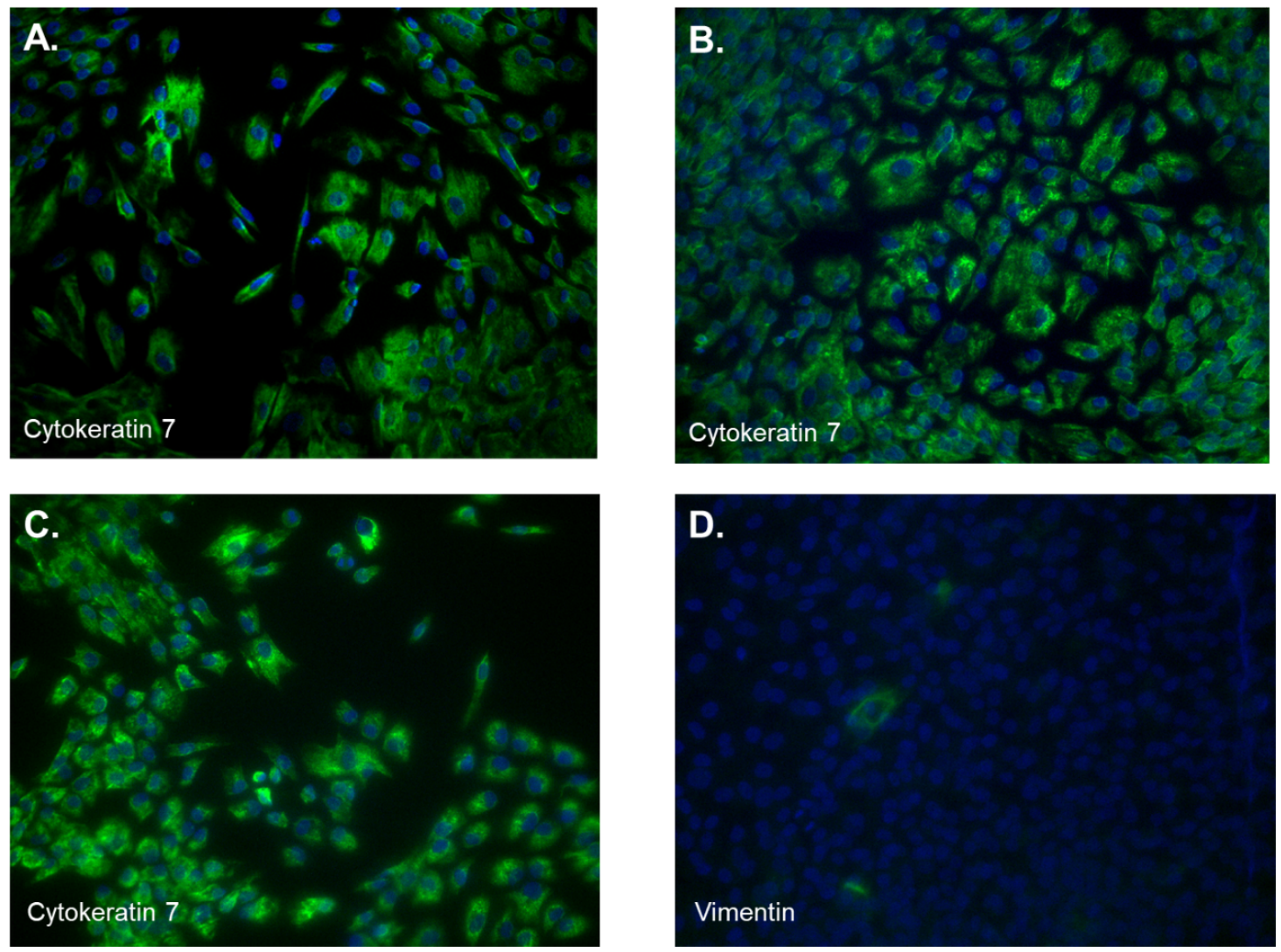

Figure 5. Immunofluorescent staining of purified trophoblasts expressing cytokeratin-7. Subcultured cytotrophoblasts are expanded in culture for 96 hours in chamber slides. (A-D); Cells stained by immunofluorescent staining with antibodies against cytokeratin 7. Nuclei were stained blue with 4',6-diamidino-2-phenylindole (DAPI). All images were obtained using a Nikon TE2000S microscope mounted with a charge-coupled device (CCD) camera at $\times 200$ magnification 


\section{IHC of primary human trophoblasts treated with forskolin for 72 hours with and without forskalin and stained for HCG}

Primary trophoblasts growth no forskolin

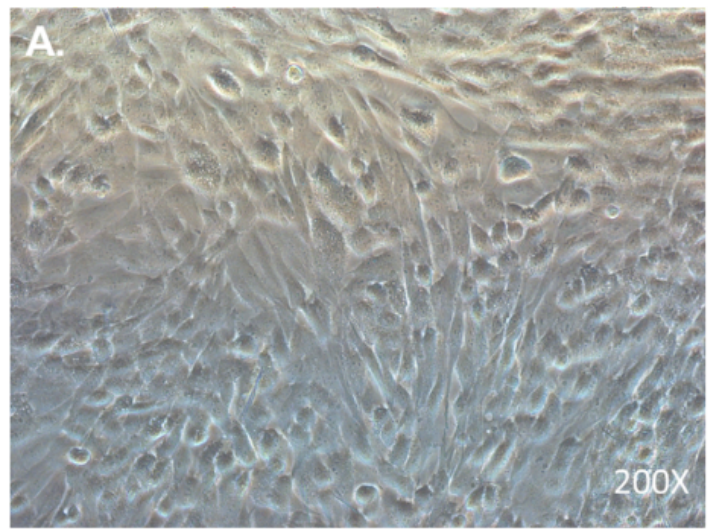

Untreated trophoblasts

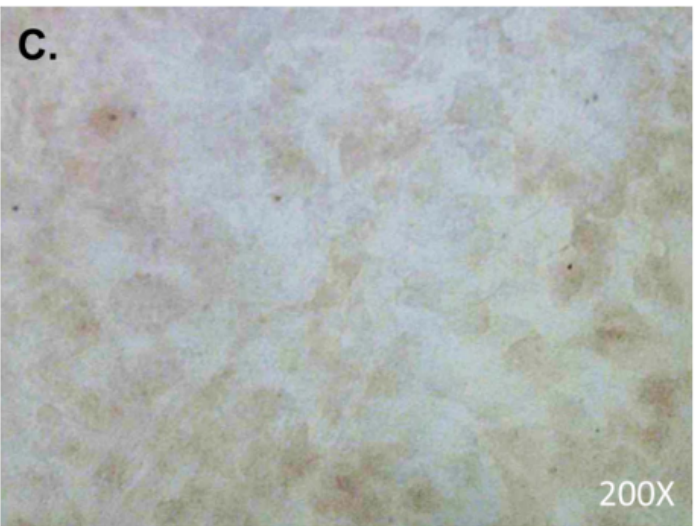

\section{Primary trophoblasts growth + forskolin}

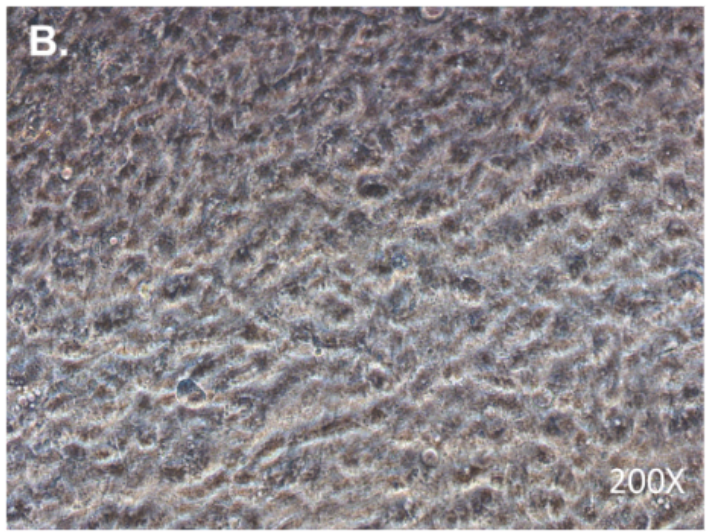

Trophoblasts treated with forskolin

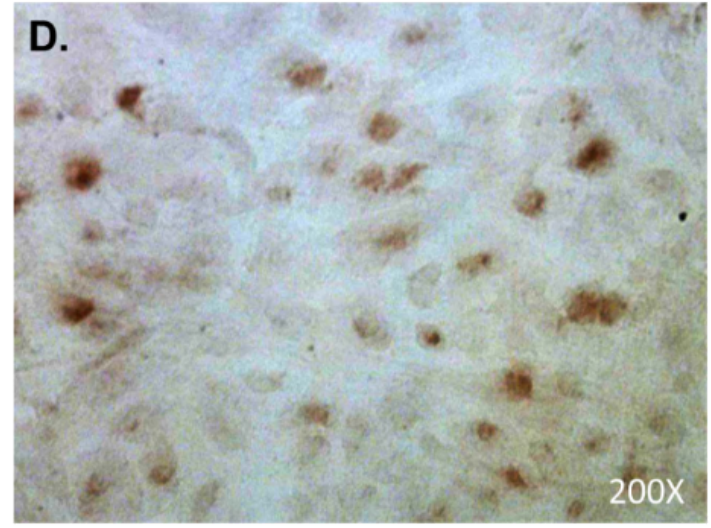

Figure 6. Cytotrophoblasts to syncytiotrophoblast transition and Immunhistochemical staining for HCG.

Subcultured cytotrophoblasts are expanded in culture for 72 hours in chamber slides to accomplish cytotrophoblast to syncytiotrophoblast transition; (A); Primary trophoblasts cultivated in chamber slides for 72 hours without forskolin; (B) Primary trophoblasts cultivated for 72 hours with forskolin; (C) Primary trophoblasts cultivated for 72 hours without forskolin and were stained immunohistochemistry with antibodies against Human HCG; (D) Primary trophoblasts cultivated for 72 hours without forskolin and were stained immunohistochemistry with antibodies against Human chorionic gonadotropin (HCG). DAB was used as a peroxidase substrate for color development. Positive trophoblasts appear brown in color. All images were obtained using a Nikon TE2000S microscope mounted with a charge-coupled device (CCD) camera at $\times 200$ magnification

and not for vimentin (mesenchymal marker) as observed in Figure 5. If trophoblasts purification techniques require tissue homogenization this will increase the likelihood endothelial cell contamination. Our procedure for trophoblast isolation does not involve tissue homogenization. This method relies of phenotyping with cytokeratin 7 and vimentin to gauge purity. Maldonado-Estrada et al., evaluated the use of Cytokeratin 7 as an accurate intracellular marker to assess the purity of human placental villous trophoblast cells by flow cytometry [25]. They demonstrate that the mutually exclusive pattern of intracellular CK7/vimentin expression of human trophoblast may be used for evaluation by flow cytometry of the purity of primary human trophoblast cells post-isolation [25]. Identification was also supported HCG expression in primary trophoblasts after treatment with Forskolin [26] (Figure 6). Several techniques have been evaluated for the isolation and purification of placental cell types mainly focused on mesenchymal stromal cells isolated from various parts of the placenta or epithelial cells isolated from amniotic membrane including cytotrophoblasts [27]. Our technique may be modified for selective isolation of cell populations from a variety of different tissues. In addition, we have used this same procedure for isolation and purification of placental fibroblasts [17]. Using this approach with multiple term placentas we have obtained reliable results. However, cultivation and yield can vary greatly if placental tissue is delayed in processing before the subculture of primary explants are performed. An important limitation to this procedure is that the initial isolation of these colonies requires extensive cultivation in vitro however, colonies will continue to develop over time (3-5 cells in the initial colony as seen in Figure 4B) and you can continue to select colonies as needed from the same placental tissue. This method is cost effective due the total amount of time a technician needs to interact with the raw tissue and the cells during cultivation. The greatest cost overall is the technicians' time and consumables.

\section{Conclusions}

In this study, I described a unique method for the isolation of cytotrophoblasts from term placenta tissue explants. This method allows for routine cultivation and purification of villous trophoblasts from placental tissue explants. Limitations exist with this approach, namely the time required for placental trophoblast outgrowth may vary 
and the rare possibility of low-level contamination with other placental cell types. This method will depend on freezing and storage of purified trophoblast populations to avoid synciatialization of cytotrophoblasts in culture over time.

\section{Author contributions}

D.J.A. is responsible for writing and reviewing the manuscript. DJA and AS performed the experiments.

\section{Funding}

D.J.A. is supported by the Meharry Zika Startup Grant and the Research Centers in Minority Institutions (RCMI) grant (U54MD007586-01). The funders did not participate in the design, preparation, data analysis, or decision to publish the manuscript.

\section{Acknowledgments}

I thank Waldemar Popik and James E.K. Hildreth for reviewing and reading the manuscript. Editing services were provided by the Meharry Office of Scientific Editing and Publications (NIH S21MD000104).

\section{Conflicts of interest}

The author declares no conflict of interest.

\section{References}

1. Mittal R, Woo FW, Castro CS, Cohen MA, Karanxha J, et al. (2019) Organ-on-chip models: Implications in drug discovery and clinical applications. J Cell Physiol 234: 8352-8380. [Crossref]

2. Lee JS, Romero R, Han YM, Kim HC, Kim CJ, et al. (2016) Placenta-on-a-chip: a novel platform to study the biology of the human placenta. J Matern Fetal Neonatal Med 29: 1046-1054. [Crossref]

3. Yin F, Zhu Y, Zhang M, Yu H, Chen W, et al. (2019) A 3D human placenta-on-a-chip model to probe nanoparticle exposure at the placental barrier. Toxicol In Vitro 54: 105113. [Crossref]

4. Blundell C, Yi YS, Ma L, Tess ER, Farrell MJ, et al. (2018) Placental drug transporton-a-chip: A microengineered in vitro model of transporter-mediated drug efflux in the human placental barrier. Adv Healthc Mater. [Crossref]

5. Pattillo RA, Gey GO (1968) The establishment of a cell line of human hormonesynthesizing trophoblastic cells in vitro. Cancer Res 28: 1231-1236. [Crossref]

6. Pattillo RA, Gey GO, Delfs E, Huang WY, Hause L, et al. (1971) The hormonesynthesizing trophoblastic cell in vitro: a model for cancer research and placental hormone synthesis. Ann N Y Acad Sci 172: 288-298. [Crossref]

7. Kohler PO, Bridson WE (1971) Isolation of hormone-producing clonal lines of human choriocarcinoma. J Clin Endocrinol 32: 683-687. [Crossref]

8. Pattillo RA, Ruckert ACF (1971) The Jar cell line -- continuous human multihormone production and controls. In Vitro 6: 398-399.

9. Kliman HJ, Nestler JE, Sermasi E, Sanger JM, Strauss 3rd JF (1986) Purification, characterization, and in vitro differentiation of cytotrophoblasts from human term placentae. Endocrinology 118: 1567e82.
10. Stenqvist AC, Chen T, Hedlund M, Dimova T, Nagaeva O, et al. (2008) An efficien optimized method for isolation of villous trophoblast cells from human early pregnancy placenta suitable for functional and molecular studies. Am J Reprod Immunol 60: 3342. [Crossref]

11. Clabault H, Laurent L, Sanderson JT, Vaillancourt C (2018) Isolation and purification of villous cytotrophoblast cells from term human placenta. Methods Mol Biol 1710 219-231. [Crossref]

12. Douglas GC, King BF (1989) Isolation of pure villous cytotrophoblast from term human placenta using immunomagnetic microspheres. J Immunol Methods 119: 259e68. [Crossref]

13. Petroff MG, Phillips TA, Ka H, Pace JL, Hunt JS (2006) Isolation and culture of term human trophoblast cells. Methods Mol Med 121: 203-217. [Crossref]

14. Huppertz B, FrankHG, Reister F, Kingdom J, Korr H, et al. (1999) Apoptosis cascade progresses during turnover of human trophoblast: analysis of villous cytotrophoblast and syncytial fragments in vitro. Lab Invest 79: 1687e702. [Crossref]

15. Guilbert LJ, Winkler-Lowen B, Sherburne R, Rote NS, Li H, et al. (2002) Preparation and functional characterization of villous cytotrophoblasts free of syncytial fragments. Placenta 23: $175 \mathrm{e} 83$.

16. Tannetta DS, Sargent IL, Linton EA, Redman CW (2008) Vitamins C and E inhibit apoptosis of cultured human term placenta trophoblast. Placenta 29: 680e90. [Crossref]

17. Aronoff DM, Correa H, Rogers LM, Arav-Boger R, Alcendor DJ (2017) Placental pericytes and cytomegalovirus infectivity: implications for HCMV placental pathology and congenital disease. Am J Reprod Immunol 78. [Crossref]

18. Alcendor DJ, Charest AM, Zhu WQ, Vigil HE, Knobel SM (2012) Infection and upregulation of proinflammatory cytokines in human brain vascular pericytes by human cytomegalovirus. J Neuroinflammation 9: 95. [Crossref]

19. Wilkerson I, Laban J, Mitchell JM, Sheibani N, Alcendor DJ (2015) Retinal pericytes and cytomegalovirus infectivity: implications for HCMV-induced retinopathy and congenital ocular disease. J Neuroinflammation 12: 2. [Crossref]

20. Pennington KA, Schlitt JM, Schulz LC (2012) Isolation of primary mouse trophoblast. J Vis $\operatorname{Exp}$ 59: e3202.

21. Kolokol'tsova TD, Saburina NN, Poltavtseva PA, Sukhikh GT (2015) Isolation and culturing of trophoblasts from human terminal placenta. Bull Exp Biol Med 158: 532536 .

22. Aboagye-Mathiesen G, Laugesen J, Zdravkovic M, Ebbesen P (1996) Isolation and characterization of human placental trophoblast subpopulations from first-trimester chorionic villi. Clin Diagn Lab Immunol 3: 14-22. [Crossref]

23. Li L, Schust DJ (2015) Isolation, purification and in vitro differentiation of cytotrophoblast cells from human term placenta. Reprod Biol Endocrinol 13: 71 [Crossref]

24. Kolokoltsova TD, Saburina IN, Zurina IM, Gorkun AA, Kosheleva NV, et al. (2017) Isolation and characterization of trophoblasts from enzymatic explants of human term placenta. Hum Cell 30: 249-257. [Crossref]

25. Maldonado-Estrada J, Menu E, Roques P, Barré-Sinoussi F, Chaouat G (2004) Evaluation of Cytokeratin 7 as an accurate intracellular marker with which to assess the purity of human placental villous trophoblast cells by flow cytometry. J Immunol Methods 286: 21-34.

26. Malhotra SS, Gupta SK (2017) Relevance of the NR4A sub-family of nuclear orphan receptors in trophoblastic BeWo cell differentiation. Cell Mol Biol Lett 22: 15.

27. Parolini O, Alviano F, Bagnara GP, Bilic G, Bühring HJ, et al. (2008) Concise review: isolation and characterization of cells from human term placenta: outcome of the first international Workshop on Placenta Derived Stem Cells. Stem Cells 26: 300-311. [Crossref]

Copyright: (C2020 Serjilus A. This is an open-access article distributed under the terms of the Creative Commons Attribution License, which permits unrestricted use, distribution, and reproduction in any medium, provided the original author and source are credited. 